\title{
Taste discrimination learning in preweanling rats
}

\author{
MARK E. STANTON \\ Environmental Protection Agency, Research Triangle Park, North Carolina \\ and University of North Carolina, Chapel Hill, North Carolina \\ and \\ MICHELLE M. NICOLLE \\ University of North Carolina, Chapel Hill, North Carolina
}

\begin{abstract}
Seventeen-day-old rat pups received intraoral infusions of two novel flavors, coffee $(0.625 \% \mathrm{w} / \mathrm{v}$ Sanka, decaffeinated) and saccharin $(0.5 \% \mathrm{w} / \mathrm{v})$, of which one (CS + ) was paired with a $0.75 \%$ body weight, i.p. injection of $0.4 \mathrm{M} \mathrm{LiCl}$, and the other (CS-) was presented alone. On the following day, two more infusion tests were conducted to determine intake of each flavor. Intake of the CS+ flavor was markedly suppressed, relative to that of the CS- flavor, although the magnitude of this effect depended on which flavor was designated CS+ and on the order in which CS+ and CS- were presented on the test day. These results indicate that preweanling rats are capable of discriminative taste aversion learning. This simple conditioning procedure should provide a useful method for studying the sensory development of the gustatory system and/or the developmental psychobiology of learning and memory.
\end{abstract}

In the 12 years since it was first demonstrated in preweanling rats (Gregg, Kittrell, Domjan, \& Amsel, 1978), taste aversion learning has become a powerful tool in the analysis of behavioral development. This learning phenomenon makes use of sensory and motor capacities that exist in infant rats, and the rapidity with which aversions are acquired facilitates age comparisons over short periods of development. These advantages have led taste aversion learning to be applied to a broad range of research problems, including developmental behavioral ecology (Gubernick \& Alberts, 1984), developmental psychopharmacology (Kehoe \& Blass, 1986), fetal behavior (Smotherman \& Robinson, 1985), interrelations between sensory and cognitive development (Rudy, Vogt, \& Hyson, 1984), infantile amnesia (Spear, 1979), and the ontogeny of stimulus selection (Spear \& Kucharski, 1984). Clearly, the adaptation of this conditioning preparation to the infant rat has contributed importantly to recent advances in our understanding of the ontogeny of learning.

During this same period, students of learning in adult animals have shown increasing interest in, and use of, dis-

\footnotetext{
This research was supported by the U.S. Environmental Protection Agency. Thanks are due Craig Barry for assistance with graphics and to Phil Bushnell and Michela Gallagher for reviewing an earlier version of this manuscript. Disclaimer: This manuscript has been reviewed by the Health Effects Research Laboratory, U.S. Environmental Protection Agency, and approved for publication. Mention of trade names or commercial products does not constitute endorsement or recommendation for use. Michelle Nicolle is now at the Curriculum in Neurobiology, University of North Carolina, Chapel Hill, NC 27514. Address correspondence to Mark E. Stanton, Neurotoxicology Division (MD-74B), U.S. Environmental Protection Agency, Research Triangle Park, NC 27711.
}

crimination learning procedures. This has occurred in part because of interest in the process of discrimination learning itself (Mackintosh, 1983; Pearce, 1987) and, in part, because discrimination designs frequently offer elegant solutions to control problems that arise in the study of other associative phenomena (e.g., Rescorla, 1985; Rescorla \& Cunningham, 1978).

Given the advantages of taste aversion for the study of learning ontogeny and the advantages of discrimination procedures for the study of learning in general, it is somewhat surprising that there have been no reports of discriminative taste aversion learning in developing rats. Recent studies of olfactory conditioning have shown that discrimination procedures may reveal learning processes that are unique to the developing rat (Spear, Kucharski, \& Miller, 1989). A procedure for studying taste discrimination learning in infant rats would make it possible to pursue similar studies in another early developing sensory system and could open the way for studies of the ontogeny of other complex associative phenomena with sophisticated within-subject designs. The purpose of this paper is to report our successful use of such a procedure and to comment on some of its methodological aspects in detail.

\section{Subjects}

\section{METHOD}

Fifteen 17-day-old Long-Evans rat pups drawn from two litters (7-8 pups per litter) were the subjects. The rats were offspring of time-bred females obtained from Charles River (Raleigh, NC) on the second day of gestation. Offspring were raised in the animal colony at the Neurotoxicology Division, U.S. Environmental Protection Agency. All subjects were maintained on a 12:12-h light:dark photoperiod (light onset at 7:00 a.m.) in plastic maternity cages measuring $48 \times 27 \times 20 \mathrm{~cm}$. 
Age of the pups was determined by checking for birth during the light phase of the light:dark cycle; the day of birth was designated Postnatal Day 0. Pups were housed with their mothers and, on the day after birth, all litters were culled to 4 males and 4 females.

\section{Design}

The subjects were trained to discriminate two flavored solutions, a $0.625 \%(w / v)$ coffee solution (Sanka, General Foods) or a $0.5 \%(w / v)$ saccharin solution (Sodium Saccharin, Fisher Scientific). On the day of conditioning, the pups received a single exposure to each flavor $4 \mathrm{~h}$ apart. Exposure to one flavor (CS+) was followed by an injection of lithium chloride ( $\mathrm{LiCl})$; the other flavor (CS-) was presented alone. A physiological saline injection was not given because, as is well known, taste aversions in infant rats cannot be attributed to the injection procedure per se (e.g., Gubernick \& Alberts, 1984; Nicolle, Barry, Veronesi, $\&$ Stanton, 1989). On the day of testing, pups were again exposed to each flavor $4 \mathrm{~h}$ apart to determine the effect of conditioning on intake of the two solutions. Test exposures were nonreinforced. The experiment was designed so as to counterbalance three factors: (1) the CS+ flavor (Sanka or Saccharin), (2) the order in which the flavors were presented on the conditioning day (CS+ first or CS- first), and (3) the order in which they were presented on the test day (CS+ first or CSfirst). The factorial combination of these variables yielded a total of eight groups. No more than one pup from a given litter was assigned to one of these groups.

\section{Procedure}

At 11:00 a.m. of Day 17, the subjects were removed from their nest and housed in individual compartments $(8 \times 9 \times 13 \mathrm{~cm})$ of a Plexiglas incubator $(37 \times 29 \times 13 \mathrm{~cm})$. The incubator contained wood shavings and was maintained at $30^{\circ}-33^{\circ} \mathrm{C}$ by an electric heating pad (General Electric) placed underneath. At this time, the pups were weighed, fitted with oral cannulas according to the procedure of Hall and Rosenblatt (1977), and returned to the Plexiglas incubator. About $5 \mathrm{~h}$ later (4:00 p.m.), the first conditioning exposure occurred. Each pup's bladder was voided by light stroking of the anogenital area with a soft artist's brush (in order to reduce inaccuracies in fluid intake measurement as a result of urination during the infusion procedure). The cannula was rinsed with $0.1 \mathrm{ml}$ of tap water to ensure it was clear and intact, the mouth and genital area were dried with a paper tissue, and the preinfusion weight of the pup was determined and recorded to the nearest $0.01 \mathrm{~g}$. The cannula was then connected to the infusion pump (Harvard, Model 906), and the pup was returned to its individual incubator compartment. The oral infusion of the appropriate flavored solution then began and continued for $15 \mathrm{~min}$ at a rate of $0.1 \mathrm{ml} / \mathrm{min}$ (yielding an infusion volume of $1.5 \mathrm{ml}$ ). After the infusion, pups were weighed, their cannulas were flushed with tap water, their mouths were towel-dried, they were (depending on group assignment) injected i.p. with $\mathrm{LiCl}(0.75 \%$ body weight, $0.4 \mathrm{M}$ solution), and they were placed in fresh incubation chambers. Intake of the solution for each pup was determined from the difference between its pre- and postinfusion weight in grams. About $4 \mathrm{~h}$ later, the second conditioning infusion began and was conducted in an identical manner, except that each pup was exposed to the alternate flavor and received the alternate drug treatment (injection vs. no injection) to that used previously. Following this second infusion, the pups were again placed in fresh individual compartments of the incubator, where they remained until testing the following day.

The first test infusion was conducted at 11:00 a.m. on the morning of Day 18 ( $24 \mathrm{~h}$ after initial deprivation). This was followed $4 \mathrm{~h}$ later by the second test infusion. These infusions were conducted in the same manner as the conditioning infusions, except that no drug was ever given. Half of the subjects were infused with the CS+ flavor first and the CSflavor second, for the other half, the order of flavor presentation was reversed.

\section{RESULTS}

Intake scores from the conditioning and test phases of the experiment were each analyzed by $2 \times 2 \times 2 \times 2$ analyses of variance (ANOVAs) involving the three between-subject factors mentioned previously (see Design section above) and the within-subject factor of stimulus (CS+ vs. CS-). During the conditioning phase, intakes of the CS+ and CS- flavors were influenced by their order of presentation (Table 1). When the CS+ flavor was presented first, consumption of the CS- flavor on the subsequent conditioning infusion was suppressed (left column of Table 1). When the CS+ flavor was presented second, consumption of the two stimuli did not differ significantly (right column of Table 1). This was reflected statistically by a main effect of stimulus $[F(1,7)=5.63, p<.05]$ and a stimulus $\times$ conditioning sequence interaction $[F(1,7)=8.69, p<.025]$. There are at least two reasons for this interaction effect. The conditioned aversion established to the CS+ flavor during the first infusion could have suppressed intake of the CS- flavor during the second infusion through stimulus generalization. Alternatively, it is possible that the $\mathrm{LiCl}$ injection paired with CS+ during the first infusion suppressed intake during the second infusion directly through an illness-induced enhancement of neophobia (Domjan, 1977). Neither of these mechanisms would operate when the CS- flavor was presented first and the CS+ flavor was presented second during conditioning. It should be noted, however, that because the flavored solutions were presented through intraoral cannulas, differential intake does not imply differential exposure to the $\mathrm{CS}+$ and $\mathrm{CS}-$ solutions during conditioning.

During the test phase of the experiment, discrimination learning was clearly evident. Mean intake of the CS+ solution was $0.152 \mathrm{ml}$, whereas intake of the CS- solution was $0.671 \mathrm{ml}$. ANOVAs of test intake scores revealed a main effect of stimulus $[F(1,7)=70.71, p<.001]$, a significant flavor $\times$ stimulus interaction $[F(1,7)=$ $16.45, p<.005$ ], and a marginally significant testsequence $\times$ stimulus interaction $[F(1,7)=4.70, p<$ $.067]$. No other statistical effects, including those involving conditioning sequence, approached significance (all $F \mathrm{~s}<1)$. The two interactions are shown, respectively, in Figures 1 and 2.

The flavor $\times$ stimulus interaction (Figure 1) appears to reflect a larger stimulus effect when saccharin served as CS+ (left panel) than when coffee served as CS+ (right panel). However, this can be attributed to differences in overall consumption of the two flavors rather than to differences in conditionability. That is, saccharin intake was lower than coffee intake regardless of whether it served as CS+ or CS- . This overestimates the stimulus effect when saccharin is CS+ and coffee is CS- , and un-

Table 1

Mean ( \pm SEM) Intake (in $\mathrm{ml}$ ) During Conditioning of the CS+ and CS- Flavors as a Function of Order of CS+ Presentation

\begin{tabular}{|c|c|c|c|c|}
\hline \multirow[b]{3}{*}{ Stimulus } & \multicolumn{4}{|c|}{ Order of CS+ Presentation } \\
\hline & \multicolumn{2}{|c|}{ First } & \multicolumn{2}{|c|}{ Second } \\
\hline & $M$ & SEM & $M$ & SEM \\
\hline $\begin{array}{l}\text { CS+ } \\
\text { CS- }\end{array}$ & $\begin{array}{l}0.45 \\
0.09\end{array}$ & $\begin{array}{l}0.12 \\
0.04\end{array}$ & $\begin{array}{l}0.36 \\
0.40\end{array}$ & $\begin{array}{l}0.08 \\
0.06\end{array}$ \\
\hline
\end{tabular}




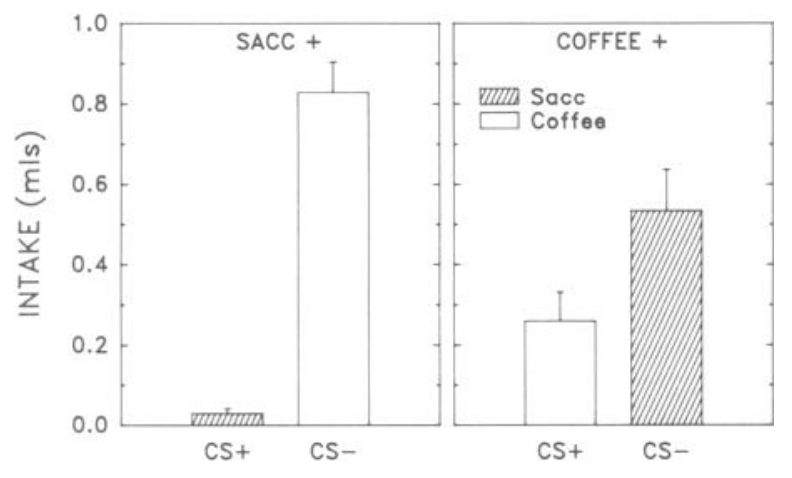

Figure 1. Mean ( \pm SEM) intake of the sacharin (SACC) and coffee (Sanka) solutions (in milliliters) during the test of discrimination learning as a function of conditioning treatment. Intake scores from the animals that received saccharin paired with $\mathrm{LiCl}$ injection (CS+) and coffee alone (CS-) appear in the left panel; scores from the animals that received the reverse treatment appear in the right panel.

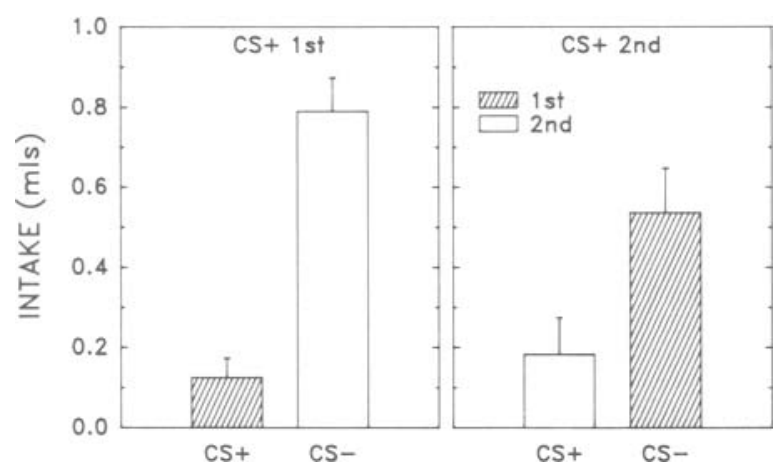

Figure 2. Mean ( \pm SEM) intake of the CS+ and CS - solutions (in milliliters) during the test of discrimination learning as a function of order of CS+ presentation during testing. Intake scores from the animals that were tested with CS+ first and CS- second appear in the left panel; scores from the animals that were tested in the reverse order appear in the right panel. Hatched bars indicate a stimulus was presented first (1st); white bars, presented second (2nd).

derestimates it in the reverse case. The stimulus effect with flavor held constant can be estimated by comparing the hatched bars (or white bars) in Figure 1 across left and right panels. The resulting difference in intake of CS+ and CS- for saccharin is about $0.51 \mathrm{ml}$, whereas this difference for coffee is about $.57 \mathrm{ml}$. Thus, the conditionability of these two flavors is very similar.

Figure 2 shows the stimulus effect (CS+ vs. CS-) as a function of the order of stimulus presentation on the test day. Conditioning appeared larger when the CS+ flavor was tested first (left panel) than when CS+ was tested second (right panel). Analogous to the flavor $X$ stimulus interaction above, this appeared to be attributable to the fact that, when the infused stimulus (CS+ or CS-) was held constant, intakes of the first infusion on the test day (hatched bars) were lower than intakes of the second in- fusion (white bars), particularly in the case of the CSflavor. There are several possible reasons for this. Intake during the first test infusion could have been suppressed through generalization of excitation or it could have been elevated during the second infusion through (generalization of) extinction. Alternatively (or in addition), thirst could have played a role, since the animals tested with CS+ first would have consumed less fluid and hence would have been thirstier at the time of the CS- infusion than would have been animals tested with CS+ second (i.e., CS- first).

\section{DISCUSSION}

The findings of this experiment indicate that preweanling rat pups can learn discriminative taste aversions involving saccharin and coffee flavors. The order in which CS+ and CS- are presented during testing can alter discrimination performance, as can assignment of flavors to stimulus condition. It is therefore important to counterbalance these two factors in studies of this kind. Discrimination performance on the test day does not, however, appear to be influenced by the order of presentation of the CS+ and CS- flavors on the conditioning day. This occurred in spite of the fact that this variable influenced conditioning intakes and suggests that it is taste exposure (which was constant) rather than flavor intake (which varied) during conditioning that is important for taste aversion learning. This confirms, in the preweanling rat, a previous observation in adult rats that, provided some intake of saccharin occurs, level of intake during oral infusions does not affect the strength of conditioned taste aversions (Domjan \& Wilson, 1972). Since the order of stimulus presentation during conditioning does not influence discrimination performance during testing, it would appear less important to counterbalance this factor. Indeed, if estimates of conditioning intakes were desired in an experiment of this kind, it would be best to always present the CS- stimulus before the CS+ stimulus. This would avoid the underestimation of CS- intake that occurs when CS+ is presented first.

This taste discrimination learning procedure has several potential applications. In addition to the study of discrimination, generalization and memory mechanisms in developing animals (e.g., Spear et al., 1989), this procedure could aid neurobiological investigations of memory development. For example, it has been proposed that maturation of gustatory neocortex contributes to the ontogeny of taste aversion learning (Vogt \& Rudy, 1984), yet the role of this brain region appears to be more prominent in discriminative taste aversion than in aversion learning involving single tastes (Kiefer \& Braun, 1977). These are just a few examples of the many problems that can be studied with the discrimination procedure reported in this article.

\section{REFERENCES}

Domun, M. (1977). Selective suppression of drinking during a limited period following aversive drug treatment in rats. Journal of Experimental Psychology: Animal Behavior Processes, 3, 66-76.

Domun, M., \& Wilson, N. E. (1972). Contribution of ingestive behaviors to taste-aversion learning in the rat. Journal of Comparative \& Physiological Psychology, 80, 403-412.

Greg,, B., Kittrell, E. M. W., Domjan, M., Amsel, A. (1978). Ingestional aversion learning in preweanling rats. Journal of Comparative \& Physiological Psychology, 92, 785-795.

GUBERNICK, D. J., ALBerTs, J. R. (1984). A specialization of taste aversion learning during suckling and its weaning-associated transformation. Developmental Psychobiology, 17, 613-628.

Hall, W. G., Rosenblatt, J. S. (1977). Suckling behavior and intake control in the developing rat pup. Journal of Comparative \& Physiological Psychology, 91, 1232-1247.

KeHOE, P., \& BLASS, E. M. (1986). Behaviorally functional opioid systems in infant rats: I. Evidence for olfactory and gustatory classical conditioning. Behavioral Neuroscience, 100, 359-367. 
KIEFER, S. W., \& BraUn, J. J. (1977). Absence of differential associative responses to novel and familiar taste stimuli in rats lacking gustatory neocortex. Journal of Comparative \& Physiological Psychology, 91, 498-507.

Mackintosh, N. J. (1983). Conditioning and associative learning. New York: Oxford University Press.

Nicolle, M. M., Barry, C. C., Veronesi, B., \& Stanton, M. E. (1989). Fornix transections disrupt the ontogeny of latent inhibition in the rat. Psychobiology, 17, 349-357.

Pearce, J. M. (1987). A model for stimulus generalization in Pavlovian conditioning. Psychological Review, 94, 61-73.

RESCORLA, R. A. (1985). Conditioned inhibition and facilitation. In R. R. Miller \& N. E. Spear (Eds.), Information processing in animals: Conditioned inhibition (pp. 299-326). Hillsdale, NJ: Erlbaum.

Rescorla, R. A., \& Cunningham, C. L. (1978). Within-compound flavor associations. Journal of Experimental Psychology: Animal Behavior Processes, 4, 267-275.

Rudy, J. W., VoGT, M. B., \& Hyson, R. L. (1984). A developmental analysis of the rat's learned reactions to gustatory and auditory stimulation. In R. Kail \& N. E. Spear (Eds.), Comparative perspectives on the development of memory (pp. 181-208). Hillsdale, NJ: Erlbaum.
Smotherman, W. P., \& Robinson, S. R. (1985). The rat fetus in its environment: Behavioral adjustments to novel, familiar, aversive and conditioned stimuli presented in utero. Behavioral Neuroscience, 99, 521-530.

SPEAR, N. E. (1979). Memory storage factors in infantile amnesia. In G. H. Bower (Ed.), The psychology of learning and motivation (Vol. 13, pp. 91-154). New York: Academic Press.

SPEAR, N. E., \& KuCHARSKI, D. (1984). Ontogenetic differences in stimulus selection during conditioning. In R. Kail \& N. E. Spear (Eds.), Comparative perspectives on the development of memory (pp. 227-252). Hillsdale, NJ: Erlbaum.

Spear, N. E., Kucharski, D., \&iller, J. S. (1989). The CS- effect in simple conditioning and stimulus selection during development. Animal Learning \& Behavior, 17, 70-82.

VoGT, M. B., \& Rudy, J. W. (1984). Ontogenesis of learning: I. Variation in the rat's reflexive and learned responses to gustatory stimulation. Developmental Psychobiology, 17, 11-33.

(Manuscript received November 4, 1989.) 